
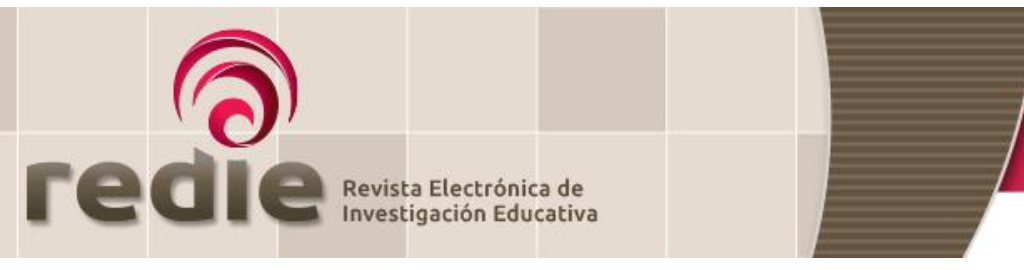

Vol. 19, Núm. 1, 2017

\title{
Transmisión pedagógica e identidad disciplinar: análisis de un caso ${ }^{1}$
}

\section{Pedagogical Transfer and Disciplinary Identity: A Case Analysis}

\author{
Gustavo Parra (*) parram@unam.mx \\ Hortensia Hickman (*) hortensiahickman@gmail.com \\ Monique Landesmann (*) segall@unam.mx \\ Miguel Ángel Pasillas (*) pasillas@unam.mx
}

(*) Universidad Nacional Autónoma de México

(Recibido: 5 de enero de 2015; Aceptado para su publicación: 3 de noviembre de 2015)

Cómo citar: Parra, G., Hickman, H., Landesmann, M. y Pasillas, M. A. (2017). Transmisión pedagógica e identidad disciplinar: análisis de un caso. Revista Electrónica de Investigación Educativa, 19(1), 98-109. Recuperado de https://doi.org/10.24320/redie.2017.19.1.1044

\section{Resumen}

El propósito de este trabajo fue analizar la propuesta de transmisión pedagógica del Curso de Formación Docente en Ciencia Básica de la carrera de Psicología en Iztacala durante el período de 1976-1985. El curso fue diseñado como circuito de reproducción de este proyecto de formación de psicólogos. Se realizaron entrevistas del itinerario de formación académica a cinco representantes de las cuatro generaciones de egresados del curso, quienes fueron elegidos para convertirse en los académicos continuadores y herederos de este modelo de formación. Se analizaron las estrategias de transmisión pedagógica del programa de formación triangulándose con los significados que los sujetos otorgaron a dichas estrategias y su impacto en la formación de la identidad académica. Los datos evidencian la función social de las prácticas de transmisión de saberes legitimados que tienen como fin el desarrollo y perfeccionamiento de los sujetos en formación, pero también la opacidad inherente a todo proyecto de formación, en términos de la apropiación y significación diferencial de los contenidos pedagógicos que se intentaban transmitir y que moldearon sus posteriores identidades como académicos.

Palabras clave: Formación docente, transmisión pedagógica, disciplina, identidad.

\section{Abstract}

The aim of this paper was to analyze the pedagogical transfer approach of the teacher training course in basic science, as part of the degree in psychology in Iztacala during the period from 1976 to 1985. The course was designed to reproduce the training project for psychologists. Interviews were conducted on the academic training of five representatives of the four

\footnotetext{
1 Este trabajo contó con el auspicio del Programa de Apoyo a Proyectos de Investigación e Innovación Tecnológica PAPIIT-EN304613 y del programa PAPCA 2013/37.
} 
graduating classes, who were picked to become the academics to inherit and continue this training model. Pedagogical transfer strategies from the training program were analyzed in a three-way connection with the meanings students gave to these strategies and their impact on the formation of an academic identity. The data reveals the social function of legitimated knowledge transfer practices that seek the development and perfection of subjects in training, but also the opacity of any training project in terms of the appropriation and differential significance of the pedagogical content that the program attempted to transmit, and which shaped their future identities as academics.

Keywords: Training project, pedagogical transmission, identity.

\section{Introducción}

El tema de la transmisión es uno de los aspectos que hay que considerar cuando se estudian las conformaciones identitarias de los sujetos en las instituciones educativas, teniendo en cuenta que en el discurso pedagógico predomina la idea de que el proceso de transmisión implica el "poner a disposición" una herencia cultural (Fattore y Caldo, 2011). En este sentido, podemos decir que todo proyecto educativo se asienta sobre procesos de transmisión, cuya intención formadora es hacer que los actores del mismo compartan saberes, prácticas, representaciones, creencias y valores, es decir, una cierta cultura. A partir de esta presunción general, presentamos los avances de una investigación que analiza la propuesta de transmisión pedagógica de un curso de formación docente, diseñado como circuito de reproducción de un proyecto de educación profesional e identidad disciplinar, a través de un estudio de caso: el Curso de Formación Docente en Ciencias Básicas (CFDCB) de la carrera de Psicología en la Escuela Nacional de Estudios Profesionales Iztacala (ENEPI) de la Universidad Nacional Autónoma de México (UNAM), entre 1976 y 1985.

En este trabajo estudiamos los procesos de transmisión mediada por la acción pedagógica de formar e instruir -es decir, los mecanismos conscientes, deliberados y planeados de la formación profesional- a través de los cuales una generación transmite una herencia de saberes y habilidades que se consideran idiosincrásicos de su profesión (Candau, 2001), presentes en el CFDCB, que los fundadores del proyecto de Psicología Iztacala diseñan para asegurar la reproducción de su modelo de formación disciplinar.

\section{Encuadre teórico-metodológico}

Aunque estos mecanismos cuidadosamente planeados para la formación docente son fundamentales como elementos objetivos de sostén de un proyecto de formación profesional, los procesos de transmisión no se reducen exclusivamente a ellos, pues es en la subjetividad donde se reelabora y resignifica aquello que se quiere transmitir. Concordamos con Frigeiro (2004) y Ferry (1990) en que la transmisión y la formación no son procesos que se den vía mecanismos transparentes en donde los sujetos se apropian de forma directa de los elementos que les son trasmitidos. Al implicar relaciones intersubjetivas, la transmisión y la formación suponen un trabajo de reelaboración por parte de los sujetos en donde siempre algo permanece y algo se transforma; "puede comprenderse como una propuesta de rasgos parciales sobre los que se lleva a cabo la posibilidad identificatoria, propia a la siempre inconclusa producción de identidad" (Frigeiro, 2004, p. 12). Esta condición fragmentaria hace necesaria una estrategia singular para poder acceder a aquello que se transmite y que es apropiado y resignificado por los sujetos.

Proponemos, con miras a develar parte de la opacidad que caracteriza a estos procesos, el análisis de las prácticas formalizadas en la propuesta educativa del CFDCB -a partir de la estrategia metodológica que se describirá más adelante-, los efectos de esta transmisión sobre los sujetos, así como los significados que éstos le asignan a su formación y conformación identitaria en torno a su disciplina, en general, y a su condición de futuros académicos de su institución, en lo particular. 
El estudio de un caso como el que aquí presentamos es, como señala Ferrarotti (citado en Mallimaci y Giménez, 2006), una síntesis horizontal de una estructura más amplia; es decir, una representación condensada de dicha estructura o mundo social (Bertaux, 2005). En este sentido, nuestro objeto de estudio contiene las lógicas de acción propias de un proyecto educativo innovador en el campo de la formación profesional, que contribuye a la conformación identitaria de los sujetos, como más adelante intentaremos demostrar.

Hemos elegido como estrategia metodológica la producción y análisis de entrevistas del relato de vida académica con egresados de este curso, en triangulación con fuentes documentales, con la finalidad de reconstruir la trama institucional que sostuvo a las dinámicas de formación y transmisión. El análisis de estos testimonios nos permitió construir los relatos de un trayecto de la vida de los sujetos desde un punto de vista etnosociológico (Berteaux, 2005), centrándonos en el recorrido académico de cada uno de ellos. Este enfoque nos permitió acercarnos tanto a la dimensión sociohistórica que encuadra los recorridos de los sujetos, en términos de los grupos familiares de origen, instituciones educativas donde se formaron y acontecimientos relevantes que marcaron su vida, como a la dimensión semántica que configura el mundo simbólico e imaginario construido por ellos. Ambas dimensiones constituyen lo que Bertaux (2005) denomina mundos sociales que encuadran el fenómeno de nuestro interés.

El presente trabajo, desde las voces de los sujetos, permite reconstruir en el itinerario biográficotemporal la versión subjetiva del fenómeno de interés, sin hacer de lado los contextos institucionales donde se despliega, para ir construyendo la semántica social de nuestro objeto; esto es, comprender su constitución, los elementos que lo configuran, las relaciones que se establecen entre los elementos, así como las prácticas recurrentes y divergentes.

Así, los testimonios fueron obtenidos a través de cinco entrevistas en profundidad del itinerario académico de alumnos egresados del CFDCB, que actualmente son académicos de tiempo completo del área experimental de la carrera de Psicología en Iztacala. Se entrevistó al único alumno de la primera generación (1976-1978), a dos de la segunda generación (1977-1979), a una de la quinta generación (1980-1982) y a uno de la séptima generación (1982-1984); cada entrevista se realizó en dos sesiones, de dos horas cada una, mismas que fueron grabadas y transcritas para facilitar su análisis. Se estableció el consentimiento informado entre el entrevistador y los entrevistados, a partir del cual autorizaron la utilización del contenido de sus relatos con fines de investigación; asimismo, cada informante recibió la transcripción de la entrevista, para que tuvieran la oportunidad de hacer precisiones, comentarios o ajustes al contenido de sus narraciones.

Mediante esta herramienta de indagación se exploraron determinados temas o procesos en el curso de la experiencia de vida del entrevistado, que consideramos significativos para los propósitos de la indagación: su trayectoria escolar, su vínculo con su disciplina, sus experiencias de formación como alumno de la licenciatura y especialmente del CFDCB, y sus prácticas de transmisión en su actual condición de docente. A partir de las perspectivas de estos protagonistas y del cruce de sus testimonios, presentamos un primer acercamiento a las etapas y aspectos destacados de nuestro objeto de estudio: los procesos de transmisión intergeneracional como parte del circuito de reproducción de un proyecto de formación disciplinar; objeto al que identificamos como una realidad compleja que muestra, desde otros ángulos, la problemática de la identidad académica que venimos trabajando desde hace más de diez años (Landesmann, Hickman y Parra, 2009).

Aunque sin la intención de hacer una sobre generalización de la representación del curso como instancia que condensa al proyecto educativo, concordamos con Saltalamacchia (1992) respecto a que "en la narración del entrevistado se pueden vislumbrar los rastros de sociabilidad que lo constituyó... [y mediante ella] es posible detectar [y] reconstruir su entorno social" (p. 120). 


\subsection{Contexto socio-institucional}

Como parte de las consecuencias del movimiento del 68, la década de los setenta del siglo XX fue un período de importantes cambios institucionales para la UNAM. Los dos rectores al frente de la institución en esa década emprendieron una serie de reformas y proyectos -aunque de distinto signo- encaminadas a transformar la vida académica de la universidad y las relaciones de ésta con la sociedad. En el rectorado del Dr. Pablo González Casanova (1970-1972) se amplió la matrícula de la enseñanza media y superior, al tiempo que se buscó elevar el nivel educativo de los estudiantes con la implantación de mejores métodos de enseñanza y nuevos contenidos educativos. Mientras que en el rectorado del Dr. Guillermo Soberón Acevedo (1973-1981) destacan las iniciativas de crecimiento institucional a partir de la creación de nuevas dependencias, que buscaban ampliar la oferta de estudios superiores mediante una estrategia de descentralización y de dar mayor impulso a la investigación.

Frente a las dificultades de introducir innovaciones en las facultades de larga tradición, las reformas se centraron en la creación de instituciones entre las que destacan el Colegio de Ciencias y Humanidades (CCH), el Sistema de Universidad Abierta (SUA) y la creación de la Escuela Nacional de Estudios Profesionales (ENEP). La génesis de nuevos espacios universitarios representó la posibilidad de ampliar la matrícula y democratizar el acceso a la universidad a sectores de clase media; así como la introducción de reformas en la organización y vida académica de la universidad, y la generación de modernos planes de estudio. Estos fenómenos, a la postre, impactaron los procesos de formación de nuevos profesionales, así como las identidades de los académicos encargados de transmitir estas propuestas.

En este marco temporal e institucional se inició la consolidación de la psicología como disciplina científica y profesional dentro de la unAM; proceso que había arrancado en la década de los sesenta con la aprobación de un plan de estudios que le daba el estatuto de profesión científica independiente (Landesmann, Hickman, Parra y Covarrubias, 2006). En 1973, los estudios de licenciatura y posgrado tuvieron un nuevo espacio universitario con la creación de la Facultad de Psicología; y en 1975, ante la creciente demanda, el H. Consejo Universitario aprobó la creación de la carrera de psicología en la Escuela Nacional de Estudios Profesionales-Iztacala (ENEP-I), en el marco de las políticas de descentralización universitaria. Pero la principal contribución de estos espacios para el desarrollo que estaba viviendo la psicología fue que hicieron posibles nuevos proyectos de formación, investigación y organización académica dentro de la UNAM.

De acuerdo con su primer director (Dr. Fernández Varela), la ENEP-ı fue el resultado "de la intersección de dos procesos que la universidad consideró necesarios: la desconcentración de los estudios superiores y la modernización de la estructura académica, expresada como la búsqueda de nuevas alternativas organizacionales y pedagógicas" (Fernández y Furlán, 1998, p. 202). En cuanto a lo educativo, señalan los mismos autores, se trataba de "construir formas pedagógicas que atenuaran o resolvieran algunos de los problemas educativos que se observaban en los planes de estudios".

La creación de la carrera de psicología en la ENEP-I (1975) se encomendó a un grupo de jóvenes universitarios, encabezados por Emilio Ribes Iñesta, quien había participado en dos importantes proyectos académicos previos: primero en la creación del Departamento de Psicología de la Universidad Veracruzana (Xalapa) entre 1964 y 1971, y después en la reorganización de la Maestría en Análisis Experimental de la Conducta, de la Facultad de Psicología de la UNAM, entre 1972 y 1974. Ambas experiencias formaron parte de un proyecto científico y profesional que intentaba desarrollar la enseñanza de esta disciplina a partir de los métodos de la psicología experimental y su aplicación a la resolución de problemas sociales.

\subsection{El proyecto de Psicología Iztacala}

Aunque la carrera de Psicología en Iztacala inició en 1975 con el plan de estudios vigente en la Facultad de Psicología -único aprobado formalmente por el H. Consejo Universitario en 1970-, 
muy pronto el equipo de Emilio Ribes se dio a la tarea de diseñar un modelo curricular a partir de un enfoque conductual y procedimientos de enseñanza derivados de la metodología del análisis experimental de la conducta (Ribes, Fernández, Talento y López,1980). Así inició el proyecto de Psicología Iztacala, como continuador del esfuerzo de consolidación de la psicología como disciplina profesional y científica, y por crear una identidad disciplinar en consonancia con este propósito. La nueva propuesta basaba su fundamentación en una severa crítica a los planes de estudio que se desarrollaban en la Facultad de Psicología de Ciudad Universitaria y se establecía como una alternativa que iba a subsanar sus deficiencias mediante nuevos modelos de enseñanza, quehacer científico y ejercicio profesional.

El currículo fue en esta época un importante dispositivo para la gestión académica, sobre todo para la innovación pedagógica. El nuevo proyecto curricular elaborado bajo el liderazgo del primer coordinador de la carrera fue "un intento por transformar profundamente nuestras concepciones y prácticas de la enseñanza superior y la ciencia y la profesión psicológica" (Ribes, 1980, p.16). El proyecto de formación profesional descansaba en el diseño de estrategias de entrenamiento para desarrollar en el alumno habilidades definidas en función de objetivos conductuales, necesarios para su intervención en el ámbito social. Es decir, se planteaba un proceso de formación que, en congruencia con el enfoque conductual, iniciaba con la determinación de las conductas que debe desarrollar un psicólogo como profesional de la disciplina y como investigador; a partir de ahí, se establecieron los objetivos educacionales mediante una secuencia de actividades de enseñanza-aprendizaje progresiva, supervisada y evaluada que se desarrollarían durante su formación. El modelo curricular adoptado fue un sistema modular constituido por situaciones de enseñanza, que especificaban actividades a realizar en tres módulos: el teórico (donde se aprendían repertorios verbales), el experimental (en el que se adquirían repertorios metodológicos) y el aplicado (en donde el estudiante aprendía a resolver problemas prácticos).

Dentro de esta lógica curricular, este modelo cobraba un lugar relevante en el ámbito de la enseñanza de la psicología en México, ya que no sólo proponía nuevas formas de hacer -enseñar, diseñar el currículo, hacer ciencia-, sino también nuevas formas de ser, es decir, implicaba la reconversión de las identidades de los estudiantes, de los docentes y de los profesionistas de la psicología.

\section{Configuración de nuevas identidades académicas}

Como se ha señalado, este modelo curricular tenía una fuerte carga utópica por su apuesta hacia el cambio no sólo de las prácticas formativas, sino también de las prácticas profesionales de los futuros psicólogos. La utopía constituye un elemento de ruptura con la historia y el pasado, contribuyendo a la renuncia -consciente o no- con las identidades previas. ${ }^{2}$ Dicha renuncia implicaba la posibilidad, para los sujetos que se inscribían en el nuevo currículo, de acceder a una identidad valorizada por un estatuto de legitimación científica. Como parte del proceso de legitimación, el modelo ponía en entredicho las deficiencias de las tradicionales prácticas curriculares que caracterizaron los sistemas de enseñanza de donde procedían la mayoría de los sujetos ya formados. Tales prácticas formativas habían constituido los referentes esenciales para la construcción de sus identidades, por lo que cuestionarlas implicaba debilitar su base identitaria. Parafraseando a Berger y Luckman (1995), se trataba de desmantelar la realidad subjetiva ya construida, para facilitar la aprehensión y apropiación adecuada de esa nueva realidad. En este sentido, Psicología Iztacala proponía un sistema de valores y normas para la disciplina, distintos a los tradicionalmente aceptados, que interpelaron a un buen número de jóvenes psicólogos en formación.

\footnotetext{
${ }^{2}$ Fernández (1998) describe cómo los fundadores de proyectos innovadores viven la etapa de puesta en marcha de los mismos. El grupo fundador "inicia un camino que muchas veces resulta despojado de todos los elementos que en la experiencia de cada uno puede ofrecer seguridad y, al modo de Hernán Cortés al quemar sus naves, hace una renuncia expresa de su propio pasado (su experiencia, la cultura de trabajo aprendida en su práctica), reniega de él y se enfrenta con un camino plagiado de peligros" (p. 208).
} 
Es decir, el proyecto educativo quedó planteado como un abierto rompimiento con lo estatuido representado por el currículum de la Facultad de Psicología-, y como modelo fundacional de un futuro distinto. Pero el rompimiento con el pasado no es fácil, el nuevo currículo tenía que instituirse sobre las herencias del viejo modelo, lo que implicaba un primer núcleo de dificultades a vencer. ${ }^{3}$ Entre ellas destaca el tipo de planta docente necesaria para esta empresa, problema que el Ribes (1980) describió así:

Los egresados de las universidades del país poseen repertorios profesionales insuficientes, tanto en lo informativo como en lo práctico, respecto a los objetivos que define el currículum de Iztacala. Por ello, de algún modo se tiene la conciencia de que, en una primera etapa, el cuerpo docente se formará paralela y conjuntamente con las primeras generaciones de egresados (p. 352)

En su etapa de arranque, el Proyecto de Psicología Iztacala tuvo que recurrir a la contratación de profesores recién egresados de su licenciatura en algunas de las escuelas de psicología que existían en el país, quienes participaron en cursos de formación docente con el objetivo de que conocieran e hicieran suyos los principios epistemológicos y educativos del proyecto. Sin embargo, esto no bastaba para construir los bastiones de la identidad académica: el vínculo con la disciplina, con el establecimiento y con la profesión (Clark, 1983).

La principal apuesta para enfrentar este problema se había diseñado desde la etapa de planeación, pues el grupo fundador preveía que la mejor manera de asegurar el éxito y la continuidad del proyecto, era reclutar a sus maestros de entre los propios alumnos egresados de Psicología Iztacala, a los que se les daría una formación especial a través de un mecanismo diseñado ex profeso: el CFDCB. Se trataba de una pieza muy importante del modelo propuesto, porque buscaba apuntalar el área neurálgica del proyecto: la formación en psicología experimental. ${ }^{4}$

\subsection{El CFDCB}

La aprobación de este curso corrió a cargo del H. Consejo Universitario, en la misma sesión en la que se aprobó el nuevo plan de estudios de orientación exclusivamente conductual, para la carrera de psicología de la ENEP Iztacala (noviembre de 1976). Las modalidades del mismo son descritas de la siguiente manera:

(...) a partir del 5o. semestre, aquellos estudiantes que habiendo cumplido con los requisitos establecidos por la Coordinación de Psicología y que con tales propósitos fueran recomendados ante el Consejo Técnico del plantel, pudieran revalidar los cuatro semestres restantes de su carrera realizando actividades de investigación básica bajo la supervisión y tutoría de especialistas en el campo de la psicología experimental. (...) Estos estudiantes toman cursos monográficos de maestría y son supervisados en docencia, instrumentación e investigación por profesores de la División de Estudios Superiores de la Facultad de Psicología de la UNAM y de la próxima a crearse en la propia ENEPI (Ribes, Fernández, Talento y López, 1980, p. 77)

Quisiéramos destacar varios aspectos de esta argumentación. Primero, la importancia simbólica que le otorga al CFDCB la propia institución, al aprobarlo junto con la nueva propuesta de

\footnotetext{
${ }^{3}$ En su trabajo de análisis sobre los proyectos de innovación educativa, Fernández (1998) describe esta etapa fundacional en los siguientes términos: "(...) en su primera concreción, que implica siempre una propuesta de organización institucional y un currículo, el grupo escribe la utopía que lo convoca y sienta la base de un mandato de exigencia que funciona como el primer motor de peligro institucional" (p. 207).

${ }^{4}$ En la etapa de arranque del Proyecto de Psicología Iztacala, el problema de contar con docentes capacitados era particularmente fuerte en el área de psicología experimental, pues sólo el 54\% de sus profesores tenían título de licenciatura; el restante $46 \%$ estaba compuesto por pasantes y alumnos con más del 75\% de los créditos de su licenciatura (Ribes, 1980, p. 76).
} 
formación profesional en psicología, como vía de formación alterna dentro del propio plan de estudio de la carrera. Esto también es una muestra de la flexibilidad institucional que se vivía en esa década, que abría paso a la innovación y a la búsqueda de alternativas de formación. Otro aspecto a destacar es el proyecto implícito de fortalecer el vínculo entre docencia e investigación, pues el curso buscaba formar académicos encargados de las labores de docencia, a partir de un fuerte entrenamiento en investigación experimental básica; con lo que además se fortalecía la intención de consolidar a la disciplina como una ciencia natural experimental y de formar psicólogos con una fuerte identidad profesional y científica. Encontramos también la intención de transformar la concepción dominante del docente universitario como profesionista liberal, para quien la actividad de enseñanza es marginal con respecto a su práctica profesional, para dar paso al académico de tiempo completo como profesional de la investigación y la enseñanza. Por último, es pertinente destacar la estrategia de formación que sus diseñadores eligieron para este curso, consistente en un esquema de supervisión y tutoría personalizada, para asegurar el correcto adiestramiento del alumno (tema sobre el que volveremos más adelante), bajo el axioma de que un psicólogo sólo podía ser formado por un verdadero psicólogo.

A pesar de la importancia estratégica del CFDCB para apuntalar el Proyecto de Psicología Iztacala, se trata de un tema poco documentado. No sólo no conocemos estudios específicos sobre esta experiencia de formación, sino que hay pocas referencias al Curso en los textos en donde los fundadores recuperan la historia de su proyecto (Ribes, 1980). Esta limitación para nuestro estudio nos llevó a elegir una estrategia metodológica basada en la producción de testimonios orales "temáticos o focalizados" (Aceves, 1999), con informantes que tuvieran un conocimiento directo del tema en cuestión.

El CFDCB formó a ocho generaciones de alumnos, con un número variable de participantes, como se muestra en la siguiente tabla:

Tabla I. Relación de las generaciones del curso de formación y el número de participantes en cada una de ellas

\begin{tabular}{lc}
\hline Generaciones & $\begin{array}{c}\text { Integrantes } \\
\text { del curso }\end{array}$ \\
\hline 1a. 1976-78 & 1 \\
2a. 1977-79 & 9 \\
3a. 1978-80 & 1 \\
4a. 1979-81 & 2 \\
5a. 1980-82 & $5^{\star}$ \\
6a. 1981-83 & 1 \\
7a. 1982-84 & 4 \\
8a. 1983-85 & 2 \\
\hline
\end{tabular}

*En esta generación, dos de los estudiantes no finalizaron su formación.

\footnotetext{
${ }^{5}$ La oferta de formación a través del CFDCB para los estudiantes elegidos tenía como culminación su inmediata contratación como profesores de la carrera. Las primeras cuatro generaciones de egresados recibieron nombramientos laborales por 40 horas y aunque esta condición de privilegio no se mantuvo para las otras generaciones, la institución mantuvo su política de contratación inmediata.
} 
Aunque era un curso formal, con aval institucional, los mecanismos de difusión para ingresar en él eran bastante informales: la Coordinación de la carrera de Psicología no publicaba abiertamente en la comunidad estudiantil las convocatorias para el ingreso, ni las características específicas de esta modalidad de formación:

Ni siquiera era una convocatoria muy difundida; sí había información de ella pero pocos la conocíamos (Estudiante de la 2a. generación)

Eran los propios maestros del área experimental los que identificaban a los alumnos destacados y los convencían de ingresar al Curso, bajo el argumento de que aprovecharan la formación de excelencia que iban a recibir:

(...) van a tener una muy buena formación, muy personalizada: son cuatro o cinco alumnos y tienes cinco o seis profesores para esos alumnos; además, muchas veces Emilio ${ }^{6}$ les da clase. Van a ser gente súper especializada en básica, en metodología (Estudiante de la 5a. generación)

Así como beneficios que a futuro se desprenderían de esta formación:

Un discurso muy común en esa época era, que si manejabas bien la ciencia básica podías hacer lo que quisieras en las ciencias aplicadas; que si tenías una buenas formación básica, una buena formación metodológica, lo ibas a hacer muy bien (Estudiante de la 5a. generación).

La composición tan reducida de alumnos de las ocho generaciones del Curso hace suponer un riguroso proceso de selección, como parte de un mecanismo que permite formar pequeños grupos de excelencia, a los que se aparta del resto de sus compañeros para darles una educación especializada; esta estrategia de formación tenía como antecedente los proyectos de innovación y experimentación pedagógica que se habían dado en algunas facultades de la UNAM a finales de la década de los sesentas, en los llamados grupos piloto. ${ }^{7}$

El proceso de selección iniciaba al finalizar el cuarto semestre del plan de estudios de la carrera, que tenía un total de ocho semestres. El alumno aspirante tenía que contar con un promedio escolar alto, no haber reprobado ninguna materia, presentar dos cartas de recomendación de sus profesores y hacer un examen de conocimientos generales. Una vez admitido al Curso hacía los cuatro semestres restantes de manera paralela al resto de la generación con la que había ingresado a la licenciatura, con la carga académica que se muestra en la tabla II:

\footnotetext{
${ }^{6}$ La cita hace referencia al Dr. Emilio Ribes, líder académico y fundador del Proyecto de Psicología Iztacala, así como diseñador del propio CFDCB.

${ }^{7}$ Es importante incluir en estos antecedentes, las experiencias de formación tutorada a grupos reducidos de alumnos, que tuvo el equipo fundador de Psicología Iztacala en el Departamento de Psicología de la Universidad Veracruzana (Xalapa) y en la Maestría en Análisis Experimental de la Conducta, de la Facultad de Psicología de la UNAM, en la década que va de 1964 a 1974.
} 
Tabla II. Plan de estudios del CFDCB

\begin{tabular}{|c|c|}
\hline 5o. semestre & 6o. semestre \\
\hline $\begin{array}{l}\text { 1. Seminario monográfico } \\
\text { 2. Seminario monográfico } \\
\text { 3. Seminario monográfico } \\
\text { 4. Seminario de Investigación e } \\
\text { Instrumentación supervisadas } \\
\text { 5. Laboratorio de investigación supervisada } \\
\text { 6. Métodos cuantitativos } \\
\text { 7. Psicología experimental (laboratorio 5o.) }\end{array}$ & $\begin{array}{l}\text { 1. Seminario monográfico } \\
\text { 2. Seminario monográfico } \\
\text { 3. Seminario monográfico } \\
\text { 4. Seminario de Investigación e } \\
\text { Instrumentación supervisadas } \\
\text { 5. Laboratorio de investigación supervisada } \\
\text { 6. Psicología experimental (laboratorio 6o.) }\end{array}$ \\
\hline 7o. semestre & 8o. semestre \\
\hline $\begin{array}{l}\text { 1. Seminario monográfico } \\
\text { 2. Seminario monográfico } \\
\text { 3. Seminario monográfico } \\
\text { 4. Seminario de Investigación e } \\
\text { Instrumentación supervisadas } \\
\text { 5. Laboratorio de investigación supervisada } \\
\text { 7. Psicología experimental (laboratorio 7o.) }\end{array}$ & $\begin{array}{l}\text { 1. Seminario monográfico } \\
\text { 2. Seminario monográfico } \\
\text { 3. Seminario monográfico } \\
\text { 4. Seminario de Investigación e } \\
\text { Instrumentación supervisadas } \\
\text { 5. Laboratorio de investigación supervisada }\end{array}$ \\
\hline
\end{tabular}

Su proceso de formación comprendía seminarios monográficos para darle una sólida preparación teórica sobre los desarrollos de la psicología conductista (adquisición de conductas verbales complejas); prácticas de laboratorio en donde se entrenaba en la investigación en ciencia básica, bajo la supervisión de un experto (adquisición de habilidades observacionales, cuantitativas y manipulativas); y práctica docente, bajo la modalidad de ayudante de un profesor titular del área experimental, donde se le adiestraba para programar y manejar su conducta en las diversas actividades que comprendían los procesos de enseñanza: principalmente, la elaboración de objetivos de entrenamiento para los estudiantes de los primeros cuatro semestres de la carrera en la materia de "Psicología experimental" y los procedimientos instruccionales adecuados para alcanzar los objetivos de esa asignatura (adquisición de complejos repertorios de interacción). Esto implicaba una carga académica para el alumno del Curso de entre seis y siete asignaturas al semestre en promedio, con un ritmo de trabajo intenso y una alta exigencia en la calidad de su desempeño, que se traducía para éste en un compromiso de formación de tiempo completo:

Teníamos un nivel de exigencia alto, era mucho material muy especializado para leer y parecía que siempre íbamos a la vanguardia. Muy especializado, muy actualizado, con mucho nivel de exigencia, que hizo que poco a poco nos fuéramos quedando a vivir aquí (...) tenías que venir sábados, domingos, días festivos, vacaciones, etc. (Estudiante de la 2a. generación)

Otro elemento que muestra el carácter atípico de esta experiencia de formación es que transcurría en un espacio intermedio entre los estudios de licenciatura y los de posgrado: estos alumnos llevaban asignaturas exclusivas del Curso, diseñadas e impartidas por algunos de los maestros del área experimental; al mismo tiempo que asistían a ciertas materias del plan general de estudios de la licenciatura, junto con los alumnos de su generación de ingreso a la carrera; así como asignaturas teóricas y de laboratorio, que cursaban con los alumnos de la Maestría de Análisis Experimental de la Conducta, de la Facultad de Psicología de la UNAM (cabe recordar que Emilio Ribes, fundador de Psicología Iztacala, participó en la creación de esta Maestría). Esta condición peculiar de su formación, que institucionalmente los distinguía como un grupo especial, sentaba las bases de una identidad colectiva que sería alimentada por la convivencia intensa y prolongada que se derivaba de sus experiencias comunes de formación.

El objetivo del Curso era entrenar a los futuros profesores en el manejo experto de aquellos repertorios teórico-prácticos que más adelante tendrían que transmitir a sus estudiantes, bajo la lógica de que sólo se puede enseñar sólidamente aquello que se demuestra con el ejemplo. Su entrenamiento temprano e intensivo estaba destinado a prepararlos en la ejecución de las 
funciones que deberían caracterizar a los profesores de Psicología Iztacala: orientación de alumnos, moderación de seminarios, supervisión de prácticas en el laboratorio, tutoría académica y diseño de objetivos instruccionales; actividades de transmisión especialmente importantes en los primeros semestres de la carrera en donde se buscaba transformar los conductas de aprendizaje con las que llegaban los estudiantes de primer ingreso.

Uno de los aspectos nodales que debería manejar el futuro docente del área experimental era el conocimiento de las labores de investigación básica, por lo que en el Curso se diseñaron prácticas de transmisión que iniciaban con su temprana incorporación en esas actividades: "El profesor debe enseñar lo que sabe hacer y no ser un simple repetidor enciclopédico de lo que otros investigadores o profesionales realizan" (Ribes et al., 1980, p. 75). La estrategia de transmisión para alcanzar este propósito descansaba en el entrenamiento tutelado, hasta que paulatinamente el estudiante pudiera plantear su propio proyecto, que sería la tesis para la titulación en la licenciatura. Ese proceso de formación en las labores de la investigación básica incluían cumplir con todos los procedimientos propios de esta actividad: el diseño de un problema de investigación en el campo de la psicología experimental, las escrupulosas prácticas en el laboratorio y la difusión de los resultados de este trabajo:

Como ya te empezabas a involucrar en el laboratorio con las cuestiones de investigación, pues es como empezar a asistir a los congresos a presentar trabajos y eso no lo hacían los estudiantes de licenciatura. Para nosotros era una obligación implícita. También estaba la presión para publicar los datos de las investigaciones (Estudiante de la 2a. generación).

Así, las prácticas de transmisión con que se diseñó el CFDCB buscaba en esencia resolver dos problemas centrales: dotar al proyecto de Psicología Iztacala de docentes con capacidad teórica y práctica para asegurar el éxito y la reproducción del modelo, y fortalecer la formación en el área experimental para formar a los futuros investigadores que se serían la base del área aplicada de la carrera de psicología.

La apuesta de transmisión de este proyecto era reforzada con la participación de connotados líderes de la psicología experimental anglosajona, quienes en calidad de profesores visitantes apuntalaban las labores de formación del CFDCB dando seminarios especializados y asesorías a los alumnos. Pero el impacto de su participación en este proyecto de transmisión rebasaba el ámbito de lo académico, para convertirse en un referente que simbolizaba la condición de privilegio de estos estudiantes, llamados a ser copartícipes en la construcción de una psicología científica en México:

Teníamos la oportunidad de escuchar de primera mano ese crisol de grandes gentes, tratarlos directamente y aprender de ellos (Estudiante de la 1a. generación)

Schoenfeld escribió un artículo en donde hablaba de los nuevos psicólogos de Latinoamérica, del grupo de jóvenes que estaba asesorando -que éramos nosotros- y que estaban construyendo un modelo muy interesante, ejemplo para Latinoamérica (estudiante de la 2a. generación)

Por lo antes expuesto, podemos decir que el CFDCB fue ideado por los constructores de Psicología Iztacala como la apuesta de transmisión más ceñida a su intención de fortalecer su proyecto de educación profesional e identidad disciplinar: un curso de formación docente para crear académicos expertos en transmitir a las nuevas generaciones de psicólogos los cuerpos de conocimientos y prácticas disciplinares, que asegurara la reproducción de su modelo de formación profesional.

\section{Conclusiones}

Desde sus inicios, Psicología Iztacala se presenta como la posibilidad única en el medio de la educación superior mexicana de ofrecer una formación basada exclusivamente en los hallazgos y 
métodos de la psicología experimental con enfoque conductual. Se trata de un proyecto de formación innovador no sólo por la orientación teórica con la que se elaboraron los contenidos, sino por las estrategias curriculares proyectadas para incidir en el núcleo central de la formación de los alumnos: la creación de un psicólogo que se identifica, define y actúa como el profesional de una ciencia. Esta conformación identitaria, aunque estará ligada a los procesos de transmisión cultural en su sentido amplio dentro del campo universitario, tendrá en las prácticas de transmisión pedagógica su apuesta más clara para consolidar y reproducir esta identidad del psicólogo; nos referimos específicamente, como se apunta al inicio de este trabajo, a los procesos de transmisión mediada por la acción pedagógica de formar e instruir, es decir, los mecanismos conscientes, deliberados y planeados de la formación profesional, a través de los cuales una generación transmite una herencia de saberes y habilidades que se consideran idiosincrásicos de su profesión.

En consonancia con el paradigma teórico adoptado, las prácticas pedagógicas de este modelo se diseñaron para reproducir condiciones de enseñanza que modificaran las conductas previas de los estudiantes. Pero, para asegurar el éxito de este proyecto sus autores advierten la necesidad de mantener una permanente vigilancia y un estricto control de las situaciones de enseñanzaaprendizaje, teniendo en cuenta que los sujetos de la formación vienen con "carencias y prácticas deformadas, heredadas por el sistema social y educativo, [que] se constituyen en barreras que se oponen al cambio" (Ribes, 1980, p. 354).

Si consideramos que la historia educacional de los sujetos determina en buena medida su comportamiento general, la progresiva y profunda transformación que pretende el proyecto tiene que ver no sólo con el contenido y las formas de aprender, sino con el desarrollo de nuevos estilos de vida. En este sentido, podemos considerar que esta apuesta de transmisión y formación buscaba la regulación normativa de discursos y modos de hacer de los sujetos, que en lo inmediato implicaba el control de su comportamiento individual a partir de un cuerpo específico de conocimientos, que le proporcionaran el marco de referencia de los comportamientos institucionalizados (Berger y Luckmann, 1968).

Esta preocupación por forjar una identidad disciplinar a partir de los procesos de transmisión pedagógica va a ser particularmente evidente en el CFDCB. Las estrategias de formación para quienes serán los herederos y continuadores del proyecto descansan en dos pilares claramente identificables: los mecanismos de selección de los sujetos, basados en el discurso de la excelencia académica; y su inclusión en un proceso educativo que sirve como laboratorio de formación, bajo la guía de quienes facilitarán al sujeto su entrenamiento en los roles que a partir de entonces tendrán estatuto de legitimidad. Los alumnos del Curso, al mismo tiempo que se preparan para ser los futuros académicos del proyecto, forman una identidad que llevan implícita una condición de distinción y compromiso, que los diferencian y apartan de sus compañeros de generación, al tiempo que los liga en una la relación iniciática, tutelar, con sus formadores.

La importancia del CFDCB no se limita a ser una apuesta innovadora en el campo de la formación docente, porque sus efectos sobre los sujetos de esa formación van más allá de los objetivos explícitos que plantea su instrucción escolarizada (en el clásico sentido de reproducción de saberes y habilidades); se trata también de un proyecto educativo que lleva implícita una intención de reencauzamiento de la trayectoria de estos sujetos, de transmisión de un nuevo ethos que valoriza de manera distinta los comportamientos apropiados, para hacer de ellos los formadores de los futuros profesionales de la psicología con una identidad distinta, vinculada a las disciplinas científicas.

A través del análisis del CFDCB podemos entender la función social de las prácticas de transmisión de saberes legitimados, que se ejerce en beneficio de un proyecto educativo, definido básicamente como "un proceso de desarrollo individual tendiente a adquirir o perfeccionar capacidades" (Ferry, 1990, p. 52). Pero también se le puede contemplar como un proceso de desarrollo y estructuración de la persona, que implica "el doble efecto de una maduración interna y de posibilidades de aprendizajes, de reencuentros y de experiencias" (Ferry, 1990, p. 53). Así 
pues, en el avance de esta investigación exploramos las estrategias y acciones educativas de quienes planearon y ejecutaron el Curso, entendidas como la intención de reencauzamiento de un formador sobre un sujeto al que se considera carente o inmaduro. A partir de estos desarrollos, nuestra investigación avanza sobre los procesos de recepción y resistencia que vivieron los sujetos de la formación durante el desarrollo estas prácticas de transmisión; pues partimos de la premisa de que en estos procesos están presentes tanto la relación que el sujeto portador de la transmisión establece con el objeto a trasmitir, como el trabajo de recepción y reelaboración de esta herencia por parte del sujeto en formación.

\section{Referencias}

Aceves, J. (1999). Un enfoque metodológico de las historias de vida. Santiago de Chile: Ediciones Sur.

Berger, P. y Luckmann, T. (1995). La construcción social de la realidad. Buenos Aires: Paidós.

Bertaux, D. (2005). Los relatos de vida. Perspectiva Etnosociológica. Barcelona: Bellaterra.

Candau, J. (2001). Memoria e identidad. Buenos Aires: Ediciones del Sol.

Fattore, N. y Caldo, P. (2011). Transmisión: una palabra clave para repensar el vínculo pedagogía, política y sociedad, en VIII Encuentro de Cátedras de Pedagogía de Universidades Nacionales Argentinas. Facultad de Humanidades y Ciencias de la Universidad Nacional de la Plata, Argentina.

Fernández, L. (1998). Crisis y dramática del cambio. Avances de investigación sobre proyectos de innovación educativa. En I. Butelman (Comp.), Pensando las instituciones. Sobre teorías y prácticas en educación. Buenos Aires: Paidós.

Ferry, G. (1990). El trayecto de la formación. Los enseñantes entre la teoría y la práctica. México: UNAM-Paidós.

Frigeiro, G. y Diker, G. (Comps.) (2004). La transmisión en las sociedades, las instituciones y los sujetos: un concepto de la educación en acción. Buenos Aires: Novedades Educativas.

Landesmann, M., Hickman, H. y Parra, G. (2009). Memorias e identidades institucionales. Fundadores y herederos en Psicología Iztacala. México: Juan Pablos.

Mallimaci F. y Giménez, V. (2006). Historias de vida y método biográfico, en Estrategias de Investigación cualitativa (pp. 175-212). Barcelona: Gedisa.

Parra, G., Hickman, H. y Segall, M. (2013). El Grupo Xalapa y la consolidación del conductismo en México: un acercamiento a la constitución de una disciplina a partir de una red intelectual. Revista Electrónica de Psicología Iztacala, 16(1), 46-64.

Ribes, E., Fernández, C., Talento, M. y López, F. (1980). Enseñanza, ejercicio e investigación de la psicología. Un modelo integral. México: Trillas.

Ribes, E. (1980). El diseño curricular en la enseñanza superior desde una perspectiva conductual: historia de un caso. En J. Urbina (Comp.), El psicólogo, ejercicio profesional y prospectiva. México: UNAM. 